\title{
DESAFIOS VIVENCIADOS PELA EQUIPE DE ATENDIMENTO PRÉ-HOSPITALAR
}

\author{
CHALLENGES EXPERIENCED BY THE PRE-HOSPITAL CARE TEAM
}

\author{
Francisco Nalécio da Costa ${ }^{1 *}$ Krysnah Allen da Silva Melo ${ }^{2} *$ Talina Carla da Silva ${ }^{3 *}$ Josefa Mayara \\ de Figueiredo Andrade ${ }^{4} *$ Jaqueline Fernandes Ribeiro $^{5}$ Jaqueline Pires Soares Hirata $^{6}$
}

\begin{abstract}
RESUMO
Introdução: $\mathrm{O}$ atendimento pré-hospitalar (APH) é definido como a assistência prestada em um primeiro nível de atenção a quadros agudos, de natureza clínica, traumática ou psiquiátrica, quando ocorrem fora do ambiente hospitalar. Porém, há entraves que dificultam a realização eficaz desse serviço como problemas relacionados ao deslocamento da ambulância, a chegada ao local da ocorrência, bem como, dificuldades durante o transporte e na chegada ao hospital. Objetivo: identificar na literatura as dificuldades vivenciadas pelos profissionais no atendimento pré-hospitalar. Método: trata-se de uma revisão integrativa, realizada em bases de dados Scielo e Lilacs, através dos descritores: assistência pré-hospitalar, ambiente de trabalho, serviços médicos de emergência, prática profissional. A busca resultou em 34 artigos, que após os critérios de inclusão: estudos científicos na íntegra, em língua portuguesa, publicados entre 2013 a 2017, de acesso livre e gratuito e que atendesse a temática, foram excluídos os que não atendiam a esses critérios, após o refinamento a pesquisa foi realizada com 03 artigos. Resultados: Fica evidente que a equipe de APH já no seu contexto laboral está exposta a riscos que favorece ao adoecimento, pois as condições de trabalho trazem locais arriscados de atuação, demandas psicológicas intensas, sobrecarga de trabalho, insatisfação e recursos insuficientes na maioria dos serviços. Conclusão: constatou-se a necessidade de buscar soluções para minimizar as dificuldades que a equipe vivência no seu cotiando, pois requer novas visões e perspectivas que possam garantir um ambiente laboral favorável e uma boa saúde do trabalhador, consequentemente uma melhor assistência.
\end{abstract}

Palavras-Chave: Assistência Pré-hospitalar Ambiente de Trabalho. Serviços Médicos de Emergência. Prática Profissional.

\begin{abstract}
Introduction: Prehospital care (PHC) is defined as the assistance provided in a first level of attention to acute clinical, traumatic or psychiatric conditions, when they occur outside the hospital environment. However, there are obstacles that impede the effective accomplishment of this service, such as problems related to ambulance movement, arrival at the place of occurrence, and difficulties during transportation and when arriving at the hospital. Objective: To identify in the literature the difficulties experienced by professionals in prehospital care. Method: It is an integrative review, carried out in Scielo and Lilacs databases, through the descriptors: prehospital care, work environment, emergency medical services, professional practice. The search resulted in 34 articles that, after the inclusion criteria: scientific studies in the Portuguese language, published between 2013 to 2017, free and free access and that addressed the theme, were excluded those that did not meet these criteria, After refinement the research was carried out with 03 articles. Results: it is evident that the APH team already in their working context is exposed to risks that favor illness, since the working conditions bring risky places of action, intense psychological demands, work overload, dissatisfaction and insufficient resources in most services. Conclusion: it was verified the need to seek solutions to minimize the difficulties that the team experiences at its rate, since it requires new visions and perspectives that can guarantee a favorable working environment and a good health of the worker, consequently a better assistance.
\end{abstract}

Keywords: Prehospital Care. Work Environment. Emergency Medical Services. Professional Practice.

\footnotetext{
${ }^{1}$ Bacharel em Enfermagem pela Faculdade Santa Maria (FSM-Cajazeiras/PB); Pós-graduado em Terapia Intensiva, Urgência/Emergência.

2 Bacharela em Enfermagem pela Faculdade Santa Maria (FSM-Cajazeiras/PB); Pós-graduada em Terapia Intensiva, Urgência/Emergência e Neonatologia; Enfermeira Dermatoterapeuta e Laserterapeuta; Enfermeira Reguladora do Hospital Regional Cleodon Carlos de Andrade (HCCA); Enfermeira plantonista da Unidade Mista Mãe Joaquina.

3 Doutora em Ciências da Saúde pela Escola de Enfermagem da Universidade de São Paulo (EEUSP)-2019. Mestre em Saúde Pública pela Universidade Estadual da paraíba (UEPB)-2014.Bacharelado e licenciatura em enfermagem formada pela Universidade Estadual da Paraíba (UEPB) 2011. Docente da Faculdade Santa Maria e Coordenadora da Pós-Graduação da Faculdade de Santa Maria de Cajazeiras. Especialista em Saúde Coletiva pela Faculdade Integrada de Patos. Cursando a Especialização de Docência no Ensino Superior pela Faculdade Santa Maria. Integrante do Núcleo de Estudos e Pesquisas Epidemiológicas (NEPE/UEPB). Membro do Grupo de Pesquisa Avaliação dos Serviços de Saúde (CNPq). Membro do Grupo de Pesquisa Vulnerabilidade, Adesão e Necessidades em Saúde Coletiva (USP). Principais Áreas de Atuação: Enfermagem, Saúde Coletiva, Epidemiologia ou Vigilância em Saúde, Doenças infectocontagiosas, Saúde Coletiva, Geoprocessamento.

${ }^{4}$ Bacharela em Enfermagem pela Faculdade Santa Maria (FSM-Cajazeiras/PB); Pós-graduada em Terapia Intensiva, Urgência/Emergência e Saúde da mulher; Enfermeira Assistencial da Maternidade Escola Assis Chateaubriand.

${ }^{5}$ Bacharel em enfermagem pelo Centro de Estudos Superiores de Maceió (CESMAC); Pós graduada em enfermagem obstétrica pela Faculdade de Educação de Bom Despacho (Pós graduada em Urgência e Emergência pela faculdade Unidas do Norte de Minas (FUNORTE); Pós graduada em UTI Geral e gestão da assistência intensiva ao paciente crítico pela Faculdade Venda Nova do Imigrante (FAVENI). Atualmente enfermeira assistencial da Maternidade Escola Assis Chateaubriand (MEAC).

${ }^{6}$ Bacharel em enfermagem pela Faculdade Santa Maria; Pós-Graduada em Enfermagem do Trabalho; e Pós-Graduada em Saúde Mental.
} 


\section{INTRODUÇÃO}

$\mathrm{O}$ atendimento pré-hospitalar (APH), segundo o Ministério da Saúde, ${ }^{1}$ pode ser definido como a assistência prestada em um primeiro nível de atenção a quadros agudos, de natureza clínica, traumática ou psiquiátrica, quando ocorrem fora do ambiente hospitalar.

Sendo assim, os serviços de APH móvel constituem importante componente da rede de atenção às urgências, cuja sua finalidade é assistir precocemente as vítimas de agravos à saúde de diversas naturezas nos locais das ocorrências, além de ser um atendimento imediato, assegura a assistência, transporte e encaminhamento às necessidades da população ${ }^{(1)}$.

Assim, para atender de maneira rápida, o APH no Brasil, é representado pelo Serviço de Atendimento Móvel de Urgência (SAMU), que foi instituído pela portaria $\mathrm{n}^{\circ} 1864 / \mathrm{GM}$, em setembro de 2003, pelo Ministério da Saúde, que estabeleceu a implantação de SAMU em municípios e regiões de todo o território brasileiro. Ampliada em 2006, passou a estabelecer regras que vão desde a especialização da equipe até as características dos veículos e os equipamentos a serem utilizados nas ambulâncias ${ }^{(2)}$.

Sua equipe é composta por múltiplos profissionais entre os quais o enfermeiro possui papel essencial na prestação do cuidado e coordenação de sua equipe, quanto suas competências, deve estar apto a supervisionar e avaliar as ações de enfermagem $^{(3)}$.

Com isso, a Portaria $\mathrm{n}^{\circ} 1.600 / 2011$, reformula a Política Nacional de Atenção às Urgências e institui a Rede de Atenção às Urgências no Sistema Único de Saúde (SUS), que tem por objetivo a ampliação do acesso e acolhimento, universalidade, equidade $\mathrm{e}$ integralidade no atendimento às urgências, como também a articulação e integração dos diversos serviços e equipamentos de saúde, atuação profissional e gestora, qualificação da assistência por meio da educação permanente das equipes de saúde do $\operatorname{SUS}^{(4)}$.

Entretanto, apesar de toda legislação existente, há alguns entraves que dificultam a realização eficaz desse serviço como problemas relacionados ao deslocamento da ambulância, a chegada ao local da ocorrência, bem como, dificuldades durante o transporte e na chegada ao hospital, conforme verificado durante a vivência desses pesquisadores.

Para o autor ${ }^{(5)}$, as dificuldades do cotidiano de trabalho se revelam durante a assistência em saúde e a questões sociais, bem como em relação ao território urbano, em suas diferentes manifestações, tais como atendimento em ruas e rodovias movimentadas, aglomerados e locais de violência. Nos quais, os profissionais estão sujeitos a mudanças climáticas, questões territoriais e sociais, defrontando-se permanentemente com o inesperado e o desconhecido. Constituindo um trabalho 
dinâmico, que é permeado pelo inesperado na realização de procedimentos e na gestão da assistência.

Porém, segundo os autores ${ }^{(6)}$ o maior desafio do atendimento pré-hospitalar se dá em relação a legislação específica tanto para os profissionais como para os atendimentos.

Diante da problemática, a pesquisa emergiu o seguinte questionamento: o que diz a literatura publicada sobre os desafios da equipe que atua em situações de urgência e emergência sofrem no seu cotidiano? Considerando a importância de identificar os desafios enfrentados pela equipe que atua nesse cenário de tensão, realizou-se este estudo com o objetivo de identificar na literatura as dificuldades vivenciadas pelos profissionais no atendimento pré-hospitalar.

\section{METODOLOGIA}

O presente estudo se trata de uma revisão integrativa, defina como aquela que condensa pesquisas anteriores e apresenta conclusões globais de um corpo de literatura especifica, permitindo a construção de análise ampla, contribuindo para discussões sobre métodos e resultados de pesquisa, resultando em uma nova aprendizagem ${ }^{(7)}$.

Corroborando, os autores ${ }^{(8)}$ afirmam que esse método de pesquisa permite a síntese de múltiplos estudos publicados e possibilita conclusões gerais a respeito de uma área de estudo abarcando analises de pesquisas relevantes que dão suporte para a tomada de decisão e a melhoria da prática clínica, possibilitando a síntese de um conhecimento novo, além de apontar lacunas do conhecimento que precisam ser preenchidas com novos estudos.

Foram percorridas seis fases para a elaboração deste estudo, sendo elas: elaboração da pergunta norteadora, busca na literatura, coleta de dados, análise crítica dos estudos incluídos, discussão dos resultados e apresentação da revisão integrativa ${ }^{(9)}$.

A pesquisa foi conduzida no mês de abril de 2020, utilizando os descritores Controlados em Ciências da Saúde (DeCS): Ambiente de trabalho, serviços médicos de emergência, assistência pré-hospitalar e prática profissional. Com esta definição, foram realizadas buscas com associações dos termos, a partir do operador booleano and, na Biblioteca Virtual de Saúde - BVS, desta forma foram selecionados artigos disponíveis na Scientific Eletronic Library Online SCIELO, Literatura Latino-Americana e do Caribe em Ciências da Saúde - LILACS. Após uma pesquisa exaustiva, obtiveram-se os seguintes resultados (figura 1): 
Figura 1: Diagrama de fluxo da seleção e inclusão dos artigos de acordo com os descritores.

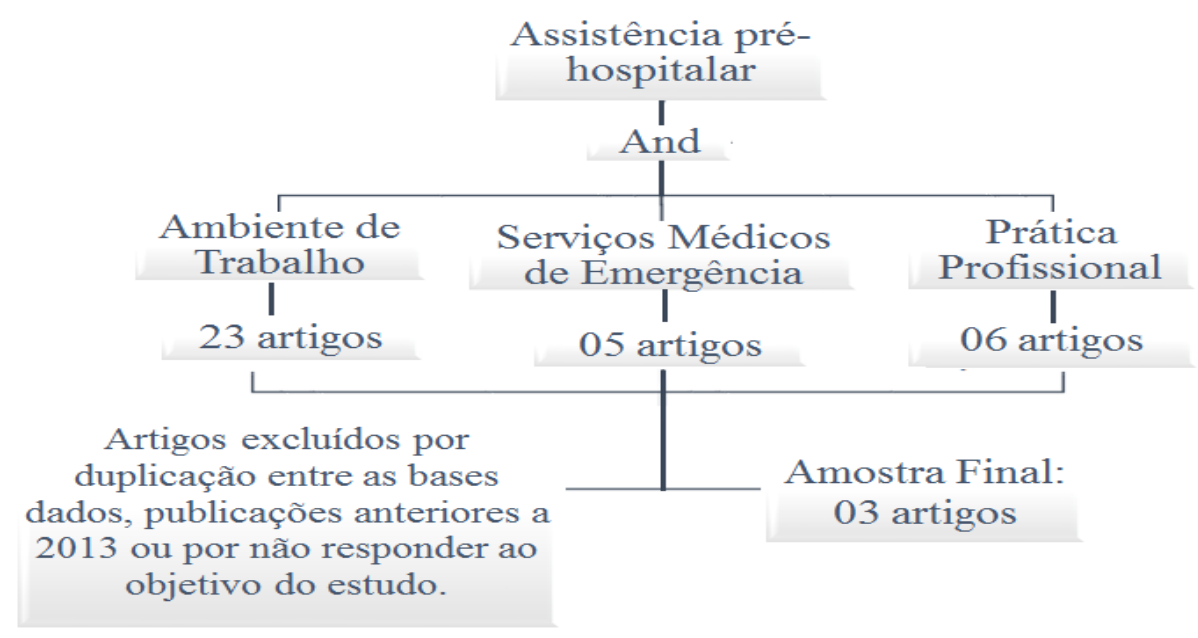

Após os critérios de inclusão e exclusão foram selecionados 03 (três) artigos potenciais. Os critérios de inclusão para a seleção dos artigos foram estudos científicos na íntegra, em língua portuguesa, publicados entre 2013 a 2020, de acesso livre e gratuito e que atendesse a temática.

Os critérios de exclusão foram desconsiderados publicações anteriores a 2013, produções não relacionadas à temática, artigos duplicados nas bases de dados, monografias, dissertações, teses e artigos indisponíveis em formato completo.

\section{RESULTADOS E DISCUSSÃO}

A produção científica sobre na linha de urgência e emergência é ampla, mas se distribuem em diversos campos e quando relacionado a equipe multiprofissional nesse setor, a literatura se difundir em estudo que abrange apenas uma categoria profissional ou a uma determinada dificuldade enfrentada por essa categoria., principalmente quando se refere aos últimos 10 anos, ficando a concentração de trabalhos contempladores da associação entre os DeCS, a quantidade de estudos diminui como se pode observar após a utilização do filtro e análise criteriosa, contemplando os critérios de inclusão delineados, chegou-se a 03 (três) artigos, os quais estavam disponíveis no SCIELO e LILACS. 
Tabela 1. Caracterização das publicações quanto ao título, aos autores, ano e resultados do estudo

\begin{tabular}{|c|c|c|c|}
\hline $\begin{array}{c}\text { SEQ. } \\
01\end{array}$ & $\begin{array}{l}\text { TÍTULO } \\
\text { Equipes e Condições de } \\
\text { trabalho nos serviços de } \\
\text { atendimento pré-hospitalar } \\
\text { móvel: revisão integrativa. }\end{array}$ & $\begin{array}{c}\text { AUTOR/ANO } \\
\text { DAL PAI et al. } \\
\text { (2015) }\end{array}$ & $\begin{array}{l}\text { RESULTADOS } \\
\text { As condições de trabalho revelam locais } \\
\text { arriscados de atuação, demandas psicológicas } \\
\text { intensas, sobrecarga de trabalho, insatisfação e } \\
\text { recursos insuficientes na maioria dos serviços. }\end{array}$ \\
\hline 02 & $\begin{array}{l}\text { Aspectos relacionados à } \\
\text { ocorrência de violência } \\
\text { ocupacional nos setores de } \\
\text { urgência de um hospital }\end{array}$ & $\begin{array}{l}\text { SOUZA, COSTA E } \\
\text { GURGEL } \\
(2014)\end{array}$ & $\begin{array}{l}\text { Constatou-se a inexistência de um protocolo } \\
\text { para orientar a equipe em casos de PCR, pois os } \\
\text { mesmos relataram agir de forma heterogênea. } \\
\text { Além disso, foi constatada a insuficiência de } \\
\text { conhecimentos sobre protocolos atuais. }\end{array}$ \\
\hline 03 & $\begin{array}{l}\text { Riscos de adoecimento } \\
\text { enfrentados pela equipe de } \\
\text { enfermagem do SAMU: } \\
\text { uma revisão integrativa }\end{array}$ & $\begin{array}{l}\text { SILVA et al. } \\
\text { (2014) }\end{array}$ & $\begin{array}{c}\text { Constatou-se que os riscos de adoecimento da } \\
\text { equipe de enfermagem do SAMU estão } \\
\text { relacionados aos fatores: físicos (ruídos e } \\
\text { temperaturas elevadas como frio ou calor), } \\
\text { químicos (contato com produtos químicos), } \\
\text { biológicos (bactérias, vírus e fungos), } \\
\text { psicológicos (estresse e agressão moral) e } \\
\text { mecânicos (acidentes automobilísticos e lesões } \\
\text { na pele). }\end{array}$ \\
\hline
\end{tabular}

Fonte: Os autores.

Para realizarmos uma análise melhor dos artigos selecionados e responder à pergunta norteadora, identificamos duas categorias: o ambiente laboral e as condições de trabalho dos profissionais de APH.

\section{Categoria 1: o ambiente laboral}

O ambiente laboral é considerado um local de risco que pode favorecer ao adoecimento, pois possui diversos fatores durante os períodos do atendimento préhospitalar como a cena do ocorrido, o transporte até o hospital e a chegada durante a vítima na instituição hospitalar ${ }^{(10)}$.

Destarte, os riscos podem ocorrer durante o desempenho dos profissionais, os quais estão expostos a bactérias e vírus, fatores climáticos, acidentes por perfuro cortantes e automobilísticos entre outros, o que pode proporcionar um ambiente de trabalho desgastante e estressante ${ }^{(10)}$.

Já o estudo dos autores ${ }^{(11)}$ enfatiza que um dos principais problemas enfrentados é quando a violência adentra o ambiente laboral e o profissional fica susceptível a ameaças, violência psicológica e agressões físicas durante o trabalho como no trajeto de ida e volta. Deixa evidente que os profissionais mais vulneráveis a violências são do sexo feminino, porém alguns profissionais têm dificuldade de agir de maneira adequada nessas situações.

$$
\text { Para os autores }{ }^{(1)} \text {, esses fatores }
$$
repercutem sobre a permanência dos 
profissionais em seus empregos bem como a saúde dos profissionais.

Contudo, os estudos revelam que o ambiente laboral já apresenta riscos para equipe de APH desde a exposição a fluidos contaminados, movimentação dos equipamentos e do paciente dentro da ambulância, mudanças climáticas, a dificuldade de acesso ao paciente, condições das rodovias, acidentes automobilísticos, dentre outros. Visto que a execução de suas atividades profissionais é num espaço púbico ficando susceptível aos "olhares curiosos", pode gerar ansiedade. E essa dificuldade ou preocupação constante pode gera estresse e adoecimento do profissional.

\section{Categoria 2: as condições de trabalho}

As condições de trabalho não consideradas favoráveis as ações laborais e nem a saúde do trabalhador, pois traz baixa remuneração, sobrecarga de trabalho e dupla jornada de trabalho ${ }^{(1,10)}$.

Outra preocupação é com a composição dessa equipe, se esses trabalhadores são habilitados ou não, cujo não há exigências em formação especifica para atender nessa área ${ }^{(1)}$.

Visto que, essa falta de experiência ou de formação específica pode ocasionar maior impacto quando enfrentarem situações estressantes ou de risco, pois não têm a mesma destreza de um profissional que atua a mais tempo no $\mathrm{APH}^{(10-11)}$.

Contudo, pode-se observar entres os artigos que a baixa remuneração pode gerar todas outras dificuldades, ou seja, se o profissional não tem uma boa remuneração ele irá ter mais de dois vínculos empregatício para ter uma boa renda familiar, mas como consequência terá sobrecarga de trabalho, que pode funcionar como um determinante de sofrimento físico e mental acaba gerando um estresse que pode refletir nas relações interpessoais e no desempenho nas atividades profissionais, como também refletir na desqualificação por não ter tempo e nem capital para educação continuada, já que em muitas empresas não são ofertadas educação permanente.

Destaca-se também, a falta de recursos humanos que serve de fator para o aumento da carga de trabalho dos profissionais existentes, outro ponto é a carência de recursos materiais, gerando baixa produtividade e qualidade do trabalho, insatisfação e desorganização do trabalho da equipe, tendem a ser desmotivante para os profissionais que apesar das dificuldades enfrentadas estão sempre dispostos a realizar um melhor trabalho, acaba levando uma relação péssima entre o trabalho e o trabalhador.

Outro fato importante, que apesar de tudo isso favorecer ao surgimento de doenças nos profissionais, os autores ${ }^{(11)}$, mostram que muitos profissionais têm dificuldade em 
aceitar que este comportamento está completamente relacionado ao trabalho realizado, traduzindo-se em um empecilho para a o aumento da visibilidade da problemática que a violência ocupacional.

\section{CONSIDERAÇÕES FINAIS}

A pesquisa constatou que o Atendimento pré-hospitalar faz parte da rede de atenção as urgências respaldo por portarias, responsável por articular os atendimentos de urgência e emergência em todo território nacional.

Foi observado que a equipe de APH é marcada por constantes desafios que resultam em oportunidades de aprendizados, mas que por outro lado pode gerar desgaste físico e emocional que deve ser discutida entre a equipe para que esses empecilhos sejam sanados, já que alguns profissionais não vêm isso como um ponto negativo, o que pode prejudicar na resolutividade do problema.

Em relação aos desafios enfrentados foi possível identificar o desgaste mental, as condições climáticas e físicas, fatores ambientais, a cena violenta, exposição a acidentes de trânsitos, violência verbal e física, são desafios que envolvem as características árduas do cotidiano da equipe. Outra questão é a exposição do enfermeiro às intempéries físicas e sociais, que compreendem elevados riscos em sua atuação, que pode ser devido os aglomerados de pessoas que se formam, já que muitos atendimentos são em rodovias. Além disso, tem os desafios profissionais diante das cenas de morte ou quase morte, conflitos nas relações profissionais, principalmente com os profissionais de serviço hospitalar que muitas vezes dificultam admissão do paciente. Outro ponto é baixa remuneração que traz como consequência vários fatores, como dupla jornada de trabalho, sobre carga de trabalho, muitas vezes até por acumular responsabilidades e profissionais desqualificados.

Trata-se de fatores que geram desmotivação dos profissionais, adoecimento como também o profissional deixe de trabalhar nesse serviço, em decorrência das pressões e das situações de trabalho enfrentadas.

Portanto, faz-se necessário a importância de boas condições de trabalho e uma remuneração adequada para o profissional buque a se qualificar para ter maior segurança nos procedimentos realizados, maior resolutividade, menor taxa de óbitos e lesões severas, além de gerar satisfação para os pacientes e equipe.

\section{REFERÊNCIAS}

1. Dal Pai D, Lima MA, Abreu KP, Zucatti PB, Lautert L. Equipes e condições de trabalho nos serviços de atendimento préhospitalar móvel: revisão integrativa. Revista Eletrônica de Enfermagem. Goiânia. v. 17, n. 4 (out./dez. 2015), p. 1-12. 2015. 
2. Brasil. Ministério da Saúde. Política Nacional de Atenção às Urgências - Portaria GM N $~^{\circ} 1863$, de 29 de setembro de 2003, Brasil. Brasília: Ministério da Saúde; 2003.

3. Campos RM. Satisfação da equipe de enfermagem do Serviço de Atendimento Móvel às Urgências (SAMU) no ambiente de trabalho (Master's thesis, Universidade Federal do Rio Grande do Norte). Natal, 2005.

4. Brasil. Ministério da Saúde. Portaria $n^{\circ}$ 1.600, de 7 de julho de 2011. Reformula a Política Nacional de Atenção às Urgências e institui a Rede de Atenção às Urgências no Sistema Único de Saúde (SUS). Brasília: Ministério da Saúde; 2011.

5. da Rocha TB. Vivências do enfermeiro no serviço de atendimento móvel de urgência: detalhes de um grande desafio. Dissertação (Mestrado em Saúde e Enfermagem) - Escola de Enfermagem, Universidade Federal de Minas Gerais, Belo Horizonte, 2013.

6. Ramos VO, Sanna MC. A inserção da enfermeria no atendimento pré-hospitalar: histórico e perspectivas atuais. Revista Brasileira de Enfermagem. 2005 Jun;58(3):355-60.

7. Dalmolin GD, Lunardi VL, Barlem EL, Silveira RS. Implicações do sofrimento moral para os (as) enfermeiros (as) e aproximações com o burnout. Texto \& ContextoEnfermagem. 2012 Mar;21(1):200-8.

8. Polit DF, Beck CT. Using research in evidence-based nursing practice. Essentials of nursing research. Methods, appraisal and utilization. Philadelphia (USA): Lippincott Williams \& Wilkins. 2006;12:457-94.

9. Souza MT, Silva MD, Carvalho RD. Revisão integrativa: o que é e como fazer. Einstein (São Paulo). 2010 Mar 1;8(1):102-6.

10. Silva OM, Ascari RA, Schiavinato D, Ribeiro MC. Riscos de adoecimento enfrentados pela equipe de enfermagem do SAMU: uma revisão integrativa. Revista de Saúde Pública de Santa Catarina. 2014 Jun 5;7(1):107-21.

11. de Souza AA, Costa WA, Gurgel AK. Aspectos relacionados à ocorrência de violência ocupacional nos setores de urgência de um hospital. Revista de Pesquisa Cuidado é Fundamental Online. 2014;6(2):637-50.

Todos os autores são afiliados da Faculdade Santa Maria, Cajazeiras - PB.

Submissão: 2020-12-01

Aprovado: 2021-04-25 